\title{
Sensor Placement for Damage Detection in Nonlinear Systems Using System Augmentations
}

\author{
Kiran D'Souza* and Bogdan I. Epureanu \\ University of Michigan, Ann Arbor, Michigan 48109-2125
}

DOI: $\underline{10.2514 / 1.33493}$

\begin{abstract}
Currently, most sensor placement methodologies are focused on maximizing the controllability and observability of the monitored structure. Recently, there have been several sensor placement techniques proposed for damage detection. The work herein provides an integrated sensor placement and reduced-order health assessment approach that can be applied to both linear and nonlinear structures. The method uses the idea that damageable regions (hot spots) of the system are often known in advance and, therefore, the modes that are sensitive to changes in these hot spots should be the ones exploited for damage detection. Generally, sensors are placed near the hot spots. However, if that is not possible or additional sensors are being used, then a generalized effective independence distribution vector method is applied for the remaining sensors. The partial eigenvector information obtained from measurements is expanded to the full (model) space using the knowledge that damage is limited to the hot spots of the system. Modalbased damage detection methods, such as minimum rank perturbation theory, can then be used to solve for the damage. Alternatively, damage identification by hot spot projection is a novel option for damage detection presented herein. Nonlinear systems are handled by forming (higher dimensional) augmented linear systems that follow the same trajectory of the nonlinear system when projected onto the physical (lower dimensional) space. The sensor placement methodology for nonlinear systems is similar, but it requires that sensors be placed at the location of all nonlinearities as well as the hot spots. Damage can be detected using the multiple augmentations generalized minimum rank perturbation theory, previously developed by the authors, or by damage identification by hot spot projection. Numerical simulations of the methodology are presented for linear and nonlinear five-bay frame structures.
\end{abstract}

\section{Nomenclature}

$\mathbf{A}_{i} \quad=$ partial eigenvalue problem

$\mathbf{B}=$ damage location matrix

c $\quad=$ time varying vector

$\mathbf{d}_{i}=$ damage location vector of the $i$ th mode

$\mathbf{E}_{i}=$ effective independence distribution vector

$\mathbf{F}=$ external excitation

$\mathbf{F}_{f}=$ filtered external excitation

$\mathbf{M}, \mathbf{K}=$ original finite element model mass and stiffness matrices

$N=$ size of full finite element model

$n=$ number of measured modes

$\mathbf{P}_{d} \quad=$ sensitive damaged modes

$\mathbf{P}_{\mathrm{dm}}=$ measured portion of sensitive damaged modes

$p=$ number of sensors

$q=$ number of damageable degrees of freedom

$\mathbf{r}=$ eigenvector sensitivity rating

$s=$ number of damage scenarios

$\mathbf{v}_{d i}=i$ th damaged eigenvector

$\mathbf{v}_{h i}=i$ th healthy eigenvector

$\mathbf{x}=$ coordinate vector

$\mathbf{x}_{f}=$ filtered coordinate vector

$\mathbf{x}_{m}=$ measured portion of the coordinate vector

$\mathbf{x}_{u}=$ unmeasured portion of the coordinate vector

$\alpha_{i}=$ level of damage in $i$ th projected damage scenario

Presented as Paper 2057 at the 48th AIAA SDM Conference, Hawaii, 1-30 April 2007; received 16 July 2007; revision received 16 June 2008; accepted for publication 19 June 2008. Copyright (C 2008 by Kiran D'Souza and Bogdan I. Epureanu. Published by the American Institute of Aeronautics and Astronautics, Inc., with permission. Copies of this paper may be made for personal or internal use, on condition that the copier pay the $\$ 10.00$ per-copy fee to the Copyright Clearance Center, Inc., 222 Rosewood Drive, Danvers, MA 01923; include the code 0001-1452/08 \$10.00 in correspondence with the CCC.

*Ph.D. Candidate, Department of Mechanical Engineering. Student Member AIAA.

${ }^{\dagger}$ Associate Professor, Department of Mechanical Engineering, Corresponding Author; epureanu@umich.edu. Member AIAA.
$\Delta \underline{\mathbf{K}}=$ perturbation to the stiffness
$\Delta \overline{\mathbf{K}}=$ projected perturbation to stiffness
$\Delta \overline{\mathbf{K}}_{i}^{\prime}=$ projected perturbation to stiffness scenarios
$\epsilon \quad=$ tolerance level
$\Lambda_{d}=$ damaged eigenvalue matrix
$\lambda_{d i}=i$ th damaged eigenvalue
$\Phi, \Psi=$ vector expansion matrices

\section{Introduction}

$\mathbf{L}$ ARGE and complex air and space structures are being placed in new and extreme conditions for extended periods of time. As a result, the need for robust and accurate health monitoring techniques continues to grow. Ideally, these health monitoring techniques would have unrestricted access to sensor information from all the degrees of freedom of a finite element model used for monitoring the integrity of the structure. Practically, however, only a limited number of locations can be instrumented due to cost, weight, and accessibility issues.

Most current sensor placement methodologies are focused on maximizing the controllability and observability of the healthy structure. For example, Cherng [1] identified the optimal placement of sensors and actuators for controllability and observability. That method examines the whole structure and selects sensor locations to maximize the signal-to-noise ratio in the system. Other approaches examine ways to minimize the information entropy norm, which is a measure of the uncertainty in parameter estimates. For example, Yuen et al. [2] proposed a sensor placement method designed for system identification and based on reducing entropy. That method requires choosing a number of damageable areas (each with an associated parameter) and placing an equal number of sensors to minimize the uncertainty in parameter estimates. Another technique, called the effective independence distribution vector (EIDV) method [3], selects sensor locations that make the measured partial eigenstructure as linearly independent as possible while capturing as much information as possible from the measured data. This method is based on capturing the dynamics of the healthy system.

Recently, several other techniques have been proposed, which are focused on sensor placement for damage detection. Cobb and Liebst 
[4] discussed one of the first such approaches. Their sensor placement technique makes no assumption about damage location, but focuses instead on a sensitivity analysis to find the degrees of freedom which maximize the changes due to damage in the observable partial eigenstructure. The method does not control which damaged components of the system are detectable. Finally, other techniques are based on maximizing the Fischer information matrix to find the optimum sensor placement for damage detection $[5,6]$. These methods make no assumption about the location of the damage; instead, they localize the damage to particular scenarios using the multiple damage location assurance criterion [7] before determining the extent of the damage.

The method herein uses a novel reduced-order modeling method combined with an eigenvector sensitivity analysis to find which eigenvectors are most sensitive to the damageable regions of interest. These damageable regions of interest are defined based on knowledge that certain regions of the system are the most likely points of damage (hot spots). This differs significantly from classical reduced-order modeling (ROM) techniques [8-14] that model the dynamics of the system and, therefore, are interested in the first few modes of the system. Instead, herein, a reduced-order health assessment (ROHA) methodology is developed to capture changes in the dynamics, which lead to different modes being of interest.

This work develops a novel sensor placement methodology specifically designed for damage detection. It places sensors at the hot spots of the system. If additional sensors need to be placed, or if certain characteristics of the hot spots of the system make placing sensors difficult or impossible, then a generalized EIDV can be formulated to place the remaining sensors.

The physical measured displacements and forcing of the structure are filtered in the frequency domain to keep only frequencies that are near the frequencies of the eigenmodes used in the projection matrix. Modal information corresponding to the measurement locations can then be extracted. This (partial) modal information can be expanded (to full modal information) by an approach which enforces that damage can only occur in the hot spots of the system. Any one of the existing modal-based damage detection methods, such as minimum rank perturbation theory [15-18] (MRPT) or optimal matrix update approaches $[19,20]$, can be used to calculate the damage. However, to provide additional noise rejection, a novel method called damage identification by hot spot projection (DIHSP) is presented in this paper.

One of the advantages of the integrated sensor placement and damage detection methodology demonstrated herein is that it can be applied to both linear and nonlinear systems if the nonlinear system can be modeled by augmented systems previously proposed by the authors $[21,22]$. These augmented systems are of higher dimension than their corresponding nonlinear systems. If the augmented systems are projected into the lower-dimension space of the nonlinear system, they will follow a single trajectory of the nonlinear system. Linear modal extraction methods can be used with augmented systems if the identification method uses a forcing that is known but not prescribed, such as direct system parameter identification [23] (DSPI) or vector backward autoregressive with exogenous modeling [24]. A linear damage detection methodology called generalized MR $\overline{P T}$ (GMRPT) has been previously developed by the authors for these augmented systems $[21,22]$.

In this work, the ROHA methodology is detailed for detecting damage in the hot spots of linear and nonlinear (augmented) systems with few measurements. Next, the methodology for an improved sensor placement is laid out for linear and nonlinear (augmented) systems. Then, the MRPT and DIHSP damage detection methodologies are explained. Finally, linear and nonlinear five-bay frames are used for various tests, illustrating the effectiveness of the proposed techniques.

\section{Methodology}

In this section, system augmentation for nonlinear systems is reviewed. Next, a novel reduced-order health assessment methodology for determining the full mode shape of linear and nonlinear (augmented) systems from partial measurement data is explained.

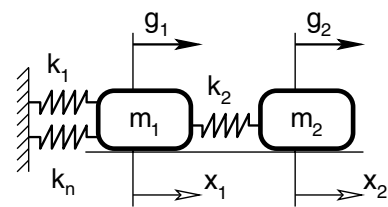

Fig. 1 Two-degree-of-freedom nonlinear mass-spring system.

Additionally, an improved sensor placement algorithm is introduced for linear and nonlinear systems when ROHA is employed. A damage detection technique based on minimum rank perturbation theory is overviewed, and then damage identification by hot spot projection is presented. Finally, filtering algorithms to reduce the effects of noise are discussed.

\section{System Augmentation}

A detailed explanation of system augmentation for nonlinear systems can be found in previous work of the authors [21,22]. In this section, a brief overview of system augmentation is explored through a simple example of a 2-degrees-of-freedom nonlinear system.

Consider a 2-degrees-of-freedom mass-spring system with one cubic nonlinearity (shown in Fig. 1). This system can be characterized by the following equations of motion:

$$
\begin{aligned}
& m_{1} \ddot{x}_{1}+\left(k_{1}+k_{2}\right) x_{1}-k_{2} x_{2}+k_{n} x_{1}^{3}=g_{1}(t) \\
& m_{2} \ddot{x}_{2}-k_{2} x_{1}+k_{2} x_{2}=g_{2}(t)
\end{aligned}
$$

where $m_{i}, k_{i}, k_{n}, g_{i}(t)$, and $x_{i}$ correspond to mass, linear spring stiffness, cubic spring stiffness, linear forcing, and displacement of the system, respectively.

The system augmentation approach consists of constructing a higher-dimensional (augmented) system that follows a single trajectory of the nonlinear system when projected down onto the lower-dimensional (physical) space. The augmented system is constructed by creating augmented variables $y_{i}$ for each nonlinearity in the system. For the system characterized by Eq. (1), only one augmented variable $y=x_{1}^{3}$ is created. Each augmented variable corresponds to 1 degree of freedom added to the system, and therefore one added equation of motion. The augmented equations of motion for Eq. (1) are

$$
\begin{aligned}
& m_{1} \ddot{x}_{1}+\left(k_{1}+k_{2}\right) x_{1}-k_{2} x_{2}+k_{n 1} y=g_{1}(t) \\
& m_{2} \ddot{x}_{2}-k_{2} x_{1}+k_{2} x_{2}=g_{2}(t) \quad m_{a} \ddot{y}+k_{c} x_{1}+k_{a} y=h(t)
\end{aligned}
$$

where $m_{a}, k_{a}, k_{c}$, and $h(t)$ correspond to the augmented mass, augmented stiffness, coupled stiffness, and augmented forcing, respectively. The parameters $m_{a}, k_{a}$, and $k_{c}$ are not unique and are chosen by the user to optimally suit their needs. The augmented forcing $h(t)$ is computed directly from the left side of Eq. (2), because $m_{a}, k_{a}$, and $k_{c}$ are chosen by the user, $x_{1}$ is measure $\bar{d}$, and $y$ is calculated by its dependence on $x_{1}$ (i.e., $y=x_{1}^{3}$ ).

The construction of the specific augmented forcing is what constrains the augmented linear system to exactly follow the nonlinear system when projected onto the lower-dimensional space (spanned by $x_{1}, x_{2}, \dot{x}_{1}$, and $\dot{x}_{2}$ ). A consequence of the augmented forcing is that modal analysis techniques that are used with augmented systems must be input-output approaches (as opposed to output-only approaches). DSPI is a multi-input/multi-output, timebased modal analysis approach that has been used successfully with augmented linear systems [21,22]. An additional consequence of the system augmentation approach is that the degrees of freedom that contain the nonlinearity must be measured to construct the augmented variable. For example, in the system given by Eq. (1), $x_{1}$ has to be measured to construct the augmented variable $y=x_{1}^{3}$.

\section{Reduced-Order Health Assessmen}

In this section, the procedure for extracting the full mode shapes that are most sensitive to damage in the hot spots of the system is outlined. 
Modal-based damage detection techniques are only effective when the modes that are used are sensitive to the damages. Reducedorder health assessment is therefore formulated to determine the full mode shapes (that are most sensitive to changes in the hot spots of the system) from limited sensor information. These damages are chosen on the basis that, in many structures, the hot spot locations are known. A sensitivity rating $\mathbf{r}$ of the eigenvectors to the $s$ different damage scenarios is evaluated as

$$
r_{i}=\sum_{j=1}^{s} \mathbf{v}_{h i}^{T} \mathbf{M} \mathbf{v}_{d i j}
$$

where $i$ denotes the eigenvector number, $j$ denotes the damage scenario, and $\mathbf{v}_{h}$ and $\mathbf{v}_{d}$ are the (augmented) eigenvectors for the healthy and damaged systems, respectively. A projection matrix that consists of the $n$ eigenvectors with the highest sensitivity (lowest $r_{i}$ ) is denoted by $\mathbf{P}$. The dimensions of $\mathbf{P}$ are $N \times n$, where $N$ is the size of the full system, and $n \ll N$.

To accurately extract the partial modes of the system corresponding to the sensitive eigenvectors, the measured forcing and positions of the system must be filtered appropriately. Consider the case where $p$ sensors measure the degrees of freedom $\mathbf{x}_{m}$ of the full model. The remaining unmeasured degrees of freedom are denoted by $\mathbf{x}_{u}$. The forcing $\mathbf{F}$ is measured. In the nonlinear case, $\mathbf{x}_{m}$ contains all the augmented variables, and $\mathbf{F}$ contains the augmented forcing. The indices of the degrees of freedom of the system are reordered such that the measured degrees of freedom of the system are first. Then, the full coordinates of the system $\mathbf{x}$ can be written as

$$
\mathbf{x}=\left[\begin{array}{l}
\mathbf{x}_{m} \\
\mathbf{x}_{u}
\end{array}\right]
$$

The modal content of $\mathbf{x}_{m}$ and $\mathbf{F}$ can be filtered by taking a Fourier transform of $\mathbf{x}_{m}$ and $\mathbf{F}$, and filtering out all frequencies except the ones near the healthy natural frequencies of the desired reduced modes. The bandwidth of the frequency filter depends on the system of interest. In particular, the changes in the frequencies due to changes in the hot spots of the system are the determining factors in the bandwidth. Because of the filtering algorithm, it is preferable to extract grouped frequencies over a specified range, rather than isolated frequencies. The filtered frequency domain data can then be returned to the time domain via an inverse Fourier transform, yielding $\mathbf{x}_{\mathrm{mf}}$ and $\mathbf{F}_{f}$, corresponding to a filtered $\mathbf{x}_{m}$ and $\mathbf{F}$, respectively. The input data of a modal analysis approach, such as DSPI, are the filtered measurements $\mathbf{x}_{\mathrm{mf}}$ and $\mathbf{F}_{f}$. DSPI is invariant to possible phase distortions caused by the inverse Fourier transform if the filtering is done similarly to both displacements and forcing. That leads to phase distortions occurring in the same manner in both the input and output used by DSPI, and, as a result, the features extracted (frequencies and partial mode shapes) are unaffected by the distortions.

The coordinates of the system can be transformed into modal coordinates as follows:

$$
\mathbf{x}=\left[\begin{array}{l}
\mathbf{x}_{m} \\
\mathbf{x}_{u}
\end{array}\right]=\sum_{i=1}^{n} c_{i}(t) \mathbf{v}_{k i}+\sum_{j=n+1}^{N} c_{j}(t) \mathbf{v}_{r j}
$$

where $\mathbf{v}_{k}$ corresponds to the kept modes of the damaged system, $\mathbf{v}_{r}$ corresponds to the removed modes of the damaged system, and $c_{i}(t)$ are time varying coefficients. The filtering process that produces $\mathbf{x}_{\mathrm{mf}}$ and $\mathbf{F}_{f}$ forces $c_{j}(t)$ to be zero for $j=n+1, \ldots, N$, which gives the following filtered response in modal coordinates:

$$
\mathbf{x}_{f}=\left[\begin{array}{c}
\mathbf{x}_{\mathrm{mf}} \\
\mathbf{x}_{u f}
\end{array}\right]=\sum_{i=1}^{n} c_{i}(t) \mathbf{v}_{k i}=\mathbf{P}_{d} \mathbf{c}
$$

where

$$
\mathbf{P}_{d}=\left[\begin{array}{lll}
\mathbf{v}_{k 1} & \mathbf{v}_{k 2} \ldots \mathbf{v}_{k n}
\end{array}\right] \quad \mathbf{c}=\left[\begin{array}{ll}
c_{1}(t) & c_{2}(t) \ldots c_{n}(t)
\end{array}\right]^{T}
$$

The output from a modal analysis approach such as DSPI is a matrix $\mathbf{P}_{\mathrm{dm}}$ which contains partial modal information, as indicated by the subscript $m$ (which corresponds to the degrees of freedom that relate to the measured ones in $\mathbf{x}$ ).

Next, one may expand each partial mode shape from $\mathbf{P}_{\mathrm{dm}}$ to the full space using the fact that damage is limited to the hot spots of the system. A damage location vector $\mathbf{d}_{i}$ used in MRPT [18], can be defined as follows (when, for example, damage only occurs in the stiffness matrix of an undamped system):

$$
\mathbf{d}_{i} \equiv\left(\lambda_{d i}^{2} \mathbf{M}+\mathbf{K}\right) \mathbf{v}_{d i}=\Delta \mathbf{K} \mathbf{v}_{d i}
$$

where $\mathbf{d}_{i}$ is the perturbation vector for the $i$ th eigenvector $\mathbf{v}_{d i}$ and natural frequency $\lambda_{d i}$. Matrices $\mathbf{M}$ and $\mathbf{K}$ are the (known) healthy mass and stiffness matrices, and $\Delta \mathbf{K}$ is the change in the healthy stiffness matrix.

The entries in $\mathbf{d}_{i}$ that correspond to degrees of freedom in the system that are undamageable (not in the hot spots) are known to be exactly zero. Therefore, if the number of measured degrees of freedom $p$ is equal to or greater than the number $q$ of degrees of freedom that are damageable, then there are at least as many equations [from Eq. (7)] as unknowns, and the inverse problem can be solved to obtain a unique solution. Two matrices $\Phi_{i}$ [of size $(N-q) \times p$ ] and $\Psi_{i}$ [of size $(N-q) \times(N-p)$ ] can be defined such that

$$
\left[\begin{array}{ll}
\Phi_{i} & \Psi_{i}
\end{array}\right]=\lambda_{d i}^{2} \tilde{\mathbf{M}}+\tilde{\mathbf{K}}=\mathbf{A}_{i}
$$

where $\tilde{\mathbf{M}}$ and $\tilde{\mathbf{K}}$ are matrices of size $(N-q) \times N$, which are composed only of the rows of $\mathbf{M}$ and $\mathbf{K}$ that correspond to the undamageable degrees of freedom. If the system is nonlinear, then the augmented degrees of freedom are not contained in $\tilde{\mathbf{M}}$ and $\tilde{\mathbf{K}}$ (even though they are undamageable). Combining Eqs. (ㄱ) and (ㅁ), one obtains

$$
\mathbf{0}=\left[\begin{array}{ll}
\Phi_{i} & \Psi_{i}
\end{array}\right]\left[\begin{array}{c}
\mathbf{v}_{m i} \\
\mathbf{v}_{u i}
\end{array}\right] \quad \mathbf{0}=\Phi_{i} \mathbf{v}_{m i}+\Psi_{i} \mathbf{v}_{u i} \quad \mathbf{v}_{u i}=-\Psi_{i}^{+} \Phi_{i} \mathbf{v}_{m i}
$$

where $\mathbf{v}_{m i}$ is the $i$ th column of $\mathbf{P}_{\mathrm{dm}}$ and represents the measured portion of the $i$ th eigenvector given by the modal analysis technique, whereas $\mathbf{v}_{u i}$ is the corresponding unmeasured portion of that eigenvector, and $\Psi_{i}^{+}$is the pseudoinverse of $\Psi_{i}$. The system is well conditioned if the number of measured degrees of freedom is equal to or greater than the number of degrees of freedom that are damageable, and $\Psi_{i}$ is full rank. One way to ensure that $\Psi_{i}$ is full rank is to properly choose the sensor locations, which is discussed next.

\section{Sensor Placement}

In this section, the sensor placement methodology is explained for linear and nonlinear systems. Of course, in the very few cases where there is exactly the same number of sensors as damageable degrees of freedom, and the sensors can be placed anywhere, they are placed at the hot spots. For the nonlinear case, sensors must be placed at the degrees of freedom that contain nonlinearities. Often, however, additional sensors may be used to reduce the effects of measurement noise. Also, some hot spots may not allow the placement of sensors nearby. In either of these cases, a generalized EIDV [3] method can be used to place the remaining sensors, as described next.

The goal of the generalized EIDV sensor placement methodology is to find the locations of sensors that lead to the largest minimum singular values of $\Psi_{i}$ for all $i$, where $\Psi_{i}$ contains $N-p$ columns of $\mathbf{A}_{i}$ in Eq. (8). Hence, EIDV [3] can be used on $\mathbf{A}_{i}$ to determine which columns of $\mathbf{A}_{i}$ contribute the least to the rank of $\mathbf{A}_{i}$, and then remove them. The procedure for EIDV is to form the matrix $\mathbf{E}_{i}$ given by [3]:

$$
\mathbf{E}_{i}=\mathbf{A}_{i}^{T}\left(\mathbf{A}_{i} \mathbf{A}_{i}^{T}\right)^{-1} \mathbf{A}_{i}
$$

Matrix $\mathbf{E}_{i}$ is an idempotent matrix with the property that its trace equals its rank. The lowest entry along the diagonal of $\mathbf{E}_{i}$ corresponds to the smallest contribution to the rank, and hence, the 
corresponding column can be removed. Matrix $\mathbf{A}_{i}$ is then recalculated without the removed column, and the process is repeated.

Because there are $n$ matrices $\mathbf{A}_{i}$ to be optimized at each step, the generalized EIDV requires $n$ matrices $\mathbf{E}_{i}$ to be formed simultaneously. Then, the entries of the diagonals of each $\mathbf{E}_{i}$ are squared. Finally, the diagonals are summed, the column corresponding to the minimum value is removed, and the process is repeated. When there are more sensors than hot spots, $\mathbf{A}_{i}$ is replaced with $\mathbf{A}_{i}^{T}$ in Eq. (10) to yield

$$
\mathbf{E}_{i}=\mathbf{A}_{i}\left(\mathbf{A}_{i}^{T} \mathbf{A}_{i}\right)^{-1} \mathbf{A}_{i}^{T}
$$

With this small adjustment, the rest of the search process is the same.

Note that the damaged natural frequencies are not known until after damage occurs. Therefore, healthy natural frequencies must be used in Eq. (8) to calculate $\mathbf{A}_{i}$ for sensor placement.

The procedure is similar for nonlinear systems modeled through augmentation. The only difference is that columns associated with the augmented degrees of freedom and the linear degrees of freedom that contain the nonlinearity are removed from $\mathbf{A}_{i}$ at the beginning of the procedure, along with the sensors that can be placed at the hot spots. This is because those degrees of freedom have to be measured to form the augmented system [21].

\section{Damage Detection Methodology: Minimum Rank Perturbation Theory}

A variety of modal-based damage detection methods can use the modes given by Eq. (9) to identify damage. In this section, minimum rank perturbation theory [18] is discussed.

MRPT was developed on the basis that damage often occurs first at localized regions of the system. Therefore, a minimum rank solution to the perturbation equations can be used. For example, for a system with damage in stiffness only (and no damping), the perturbation equations are defined in Eq. (7). The minimum rank solution to the perturbation equations for $\bar{\Delta} \mathbf{K}$ (using MRPT and a subspace selection algorithm) is given by [18]

$$
\Delta \mathbf{K}=\mathbf{B Z}\left(\mathbf{Z}^{T} \mathbf{B}^{T} \mathbf{P}_{d} \mathbf{Z}\right)^{-1} \mathbf{Z}^{T} \mathbf{B}^{T}
$$

where $\mathbf{B}=\left[\begin{array}{ll}\mathbf{d}_{1} & \mathbf{d}_{2} \cdots \mathbf{d}_{n}\end{array}\right], \mathbf{Z}=\mathbf{V} \Sigma_{\epsilon}, \mathbf{B}=\mathbf{U} \Sigma \mathbf{V}^{T}$, and $\mathbf{U}, \Sigma$, and $\mathbf{V}$ form the standard singular value decomposition of matrix $\mathbf{B}$, whereas $\Sigma_{\epsilon}$ contains the singular values that are greater than a tolerance level $\epsilon$

If the system is an augmented one, damage is first calculated in the nonlinear parameters using the multiple augmentations approach developed by the authors [22].

\section{Damage Detection Methodology: Damage Identification by Hot Spot Projection}

This section introduces an alternate damage detection methodology (to MRPT) called damage identification by hot spot projection. This approach has been developed on the basis that damage is constrained to a linear combination of $s$ possible damage scenarios (or hot spots).

The eigenvalue problem for $n$ modes of the damaged system can be written as

$$
\mathbf{M} \mathbf{P}_{d} \Lambda_{d}^{2}+\mathbf{K} \mathbf{P}_{d}=\Delta \mathbf{K} \mathbf{P}_{d}
$$

where $\Lambda_{d}=\operatorname{diag}\left[\begin{array}{ll}\lambda_{d 1} & \lambda_{d 2} \cdots \lambda_{d n}\end{array}\right]$. Equation (13) can be projected onto a reduced space by premultiplying it by $\mathbf{P}_{d}^{T}$ to obtain reducedorder matrices $\Delta \overline{\mathbf{K}}$ as

$$
\mathbf{P}_{d}^{T} \mathbf{M} \mathbf{P}_{d} \Lambda_{d}^{2}+\mathbf{P}_{d}^{T} \mathbf{K} \mathbf{P}_{d}=\mathbf{P}_{d}^{T} \Delta \mathbf{K} \mathbf{P}_{d}=\Delta \overline{\mathbf{K}}
$$

Using the knowledge that only certain regions of the system are damageable, different $\Delta \overline{\mathbf{K}}_{i}^{\prime}$ can be generated as a set of basis matrices for damage in the reduced space. A requirement of these basis matrices is that they are linearly independent. These basis matrices are calculated by transforming the physical damage scenarios to the reduced ones using the following relation:

$$
\mathbf{P}_{d}^{T} \Delta \mathbf{K}_{i} \mathbf{P}_{d}=\Delta \overline{\mathbf{K}}_{i}^{\prime}
$$

where $\Delta \mathbf{K}_{i}$ is the damage in the stiffness matrix in the full (augmented) space, and $i=1, \ldots, s$ corresponds to the damage scenario. The damage predicted can then be written as

$$
\Delta \overline{\mathbf{K}}=\sum_{i=1}^{s} \Delta \overline{\mathbf{K}}_{i}^{\prime} \alpha_{i}
$$

where $\alpha_{i}$ corresponds to the level of damage corresponding to the $i$ th damage scenario.

If the matrices $\Delta \overline{\mathbf{K}}_{i}^{\prime}$ from Eq. (16) are transformed into column vectors by stacking the columns of each matrix on top of each other, vectors of size $n^{2}$ will result. When $n^{2}>s$ and the $\Delta \overline{\mathbf{K}}_{i}^{\prime}$ matrices are independent, an overdetermined set of equations results for calculating the damage, and $\alpha_{i}$ can be obtained from Eq. (16) (from the computed $\Delta \overline{\mathbf{K}}_{i}^{\prime}$ and measured $\Delta \overline{\mathbf{K}}$ ).

\section{Filtering Algorithms}

There are two filtering algorithms that can be used with the DIHSP and MRPT methodologies to reduce the effects of noise. The first filtering algorithm uses the fact that different damage scenarios affect the natural frequencies of the system differently. This filtering can be implemented by first determining which natural frequencies are changed significantly by each damage scenario (in the damage range of interest). After damage occurs, the natural frequencies can be inspected to identify which ones were affected. Finally, any damage scenario that would cause a change in a natural frequency of the system that remains unaffected is eliminated as a possible damage scenario. This information can be used in Eq. (15). Essentially, basis matrices $\Delta \overline{\mathbf{K}}_{i}^{\prime}$ do not have to be computed for the eliminated damage scenarios, which filters out any damage that would be erroneously predicted in that space (due to measurement noise). This filtering algorithm is particularly powerful for cases where damages occur in only a few damage scenarios.

The second filtering algorithm is based on the fact that the minimum singular values of the different $\Psi_{i}$ matrices can be different in scale. A threshold value can be used such that if the minimum singular value for a given $i$ is lower than the threshold, then that $\Psi_{i}$ would not be used (in turn, the eigenvector it corresponds to would not be calculated). This filtering algorithm is important because the singular values of $\Psi_{i}$ are not known until after damage occurs (and the natural frequencies of the damaged system can be measured).

\section{Numerical Results}

To demonstrate the proposed methodology, a numerical analysis of linear and nonlinear frame structures was implemented. The linear frame structure shown in Fig. 2 consists of 70 steel beams connected at 24 nodes, four of which are pinned to the ground. The elastic modulus of the steel used is $200 \mathrm{GPa}$ and its density is $7845 \mathrm{~kg} / \mathrm{m}^{3}$. The length of the entire structure is $2.5 \mathrm{~m}$, whereas the width and height of the entire structure are $0.5 \mathrm{~m}$. The cross section of each individual beam is a $30-\mathrm{mm}$-wide, hollow square with a $1 \mathrm{~mm}$ wall

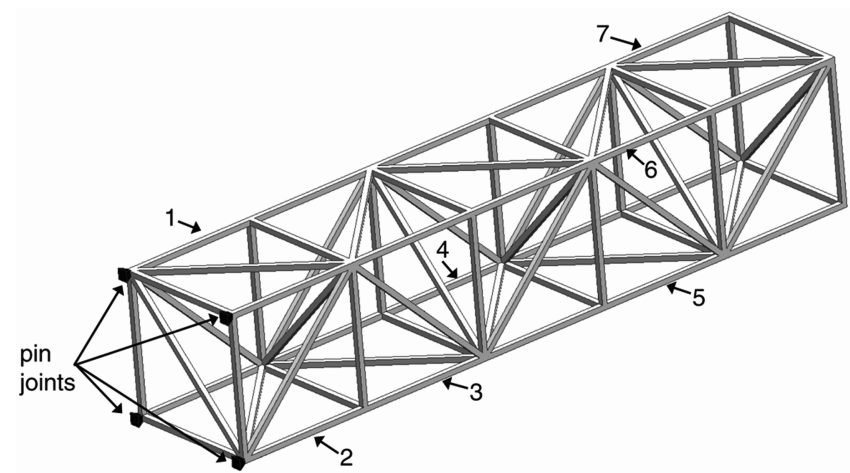

Fig. 2 Linear five-bay frame structure. 


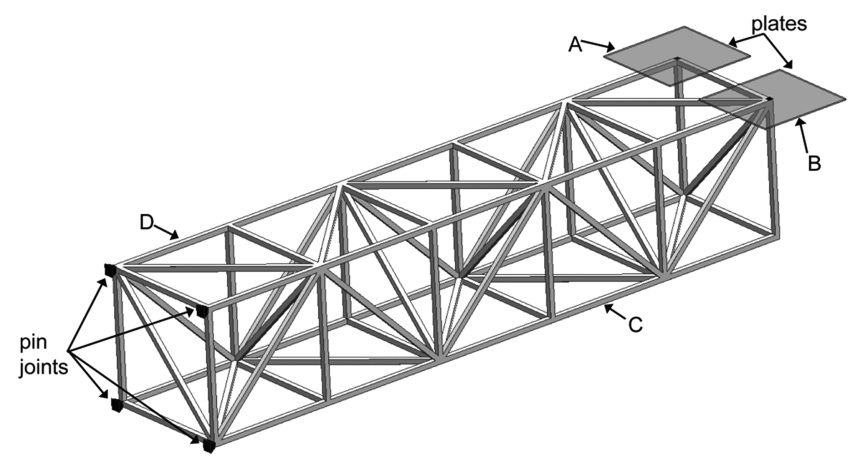

Fig. 3 Linear five-bay structure with two plates which introduce cubic stiffness nonlinearities.

thickness. The damageable portions of the linear system were chosen as the transverse (bending) stiffnesses of the 20 longitudinal beams. A finite element model for the structure was constructed using five beam elements to discretize each of the beams that are not aligned in the longitudinal (horizontal) direction, for a total of $1332 \mathrm{deg}$ of freedom. To verify that this discretization is converged, the frequencies and mode shapes for this model were compared with those given by a much more refined model, where 20 beam elements were used to discretize each and every beam in the structure. The first 50 frequencies and mode shapes for the two models were in very good agreement.

The nonlinear frame structure shown in Fig. 3 consists of the same linear frame structure as in Fig. 2 with the addition of two plates connected to the frame at their center. These plates are pinned to the ground at their perimeter. Hence, they exhibit stretching induced by bending. Using a one-mode Galerkin approximation for each plate, a linear and cubic stiffness is introduced to the frame structure at the point of attachment. The characteristics of the plates are such that the stiffness of the cubic spring nonlinearity added to the system by each plate is $60 \mathrm{MN} / \mathrm{m}^{3}$. The damageable portions of the nonlinear system were chosen as the transverse stiffness of the 20 longitudinal beams and the two plates. The full augmented linear system has a total of 1334 deg of freedom.

In the following sections, several important aspects of the novel methodology are highlighted. First, the differences between ROM and ROHA are explored. Second, a comparison case is setup for MRPT and DIHSP. Then, the robustness of the algorithm for damage outside of the hot spots is explored. Next, the effects of the filtering algorithms and the placement of additional sensors are illustrated. Finally, damage cases for the nonlinear frame system are examined.

Sensors were placed at the hot spots for all the results obtained. In the linear system, that implies that the sensors measure the transverse displacement of the 20 longitudinal beams. The nonlinear system has the same 20 sensors as the linear system.

\section{Reduced-Order Health Assessment Versus Reduced-Order Modeling}

This section highlights the differences between a ROM-type method and ROHA. ROM is designed to predict the system dynamics. In contrast, ROHA is designed to predict changes in the system dynamics. ROM uses the first (dominant) several modes of the system to capture the dynamics of the system. This fundamentally differs from ROHA, which uses the eigenvectors sensitive to changes in the hot spots of the system. In contrast to a usual ROM, in this work, the modes chosen for ROHA were the 11th-30th.

Figure 4 illustrates the difference in applying the ROM method and using the first 20 modes as opposed to more sensitive modes obtained by using ROHA and Eq. (3). The case plotted in Fig. 4 is for a $15 \%$ loss of transverse stiffness in beam 2 , and a $20 \%$ loss of transverse stiffness in beam 6 , where these beam numbers are shown in Fig. 2. Standard deviation error bars are plotted for the 100 separate numerical simulations in which $10 \%$ random eigenvector noise was added. The noise was added into the partial eigenstructure. The $x$ axis in each plot represents the 20 damage scenarios (i.e., the 20 transverse stiffnesses of the 20 longitudinal beams). The $y$ axis in

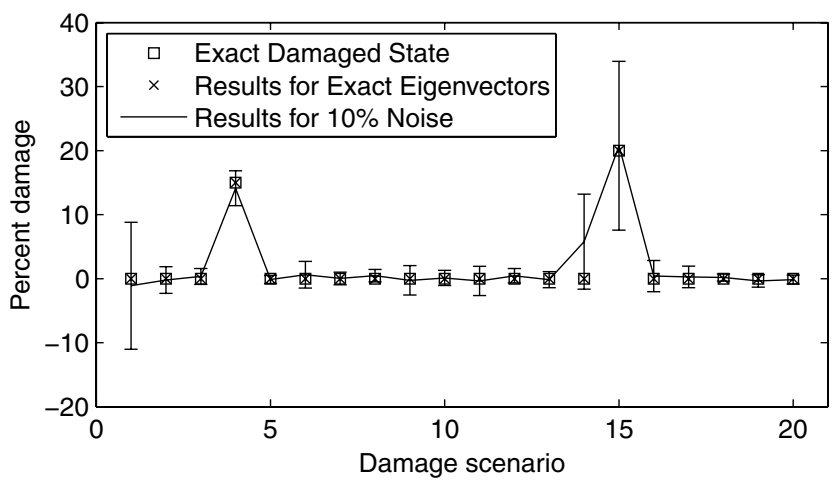

a) ROM

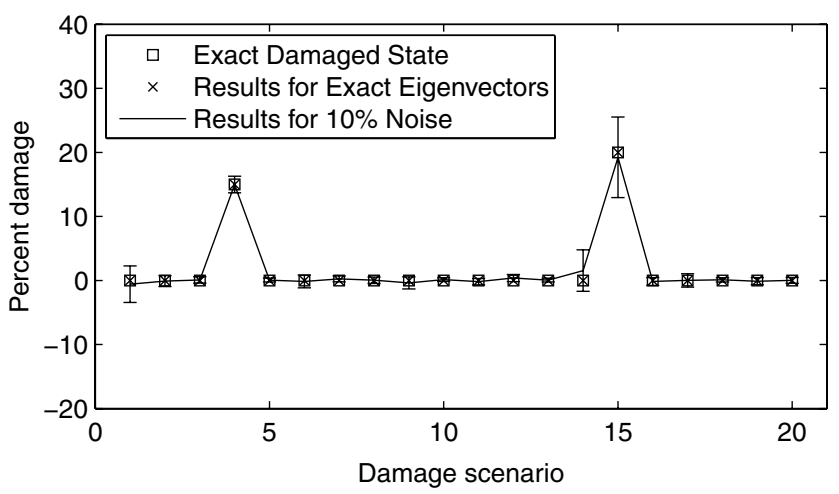

b) ROHA

Fig. 4 Predicted damage in the linear five-bay frame with damage at two locations and $10 \%$ random eigenvector noise using a) the first 20 modes of the system and b) 20 sensitive modes to damage in the hot spots.

each plot represents the percent damage for each scenario. No filtering algorithm was applied for this case, and 20 sensors were used. DIHSP was the damage methodology used to obtain the results. Figure 4a shows results obtained using the dominant modes of the system, whereas Fig. $4 \mathrm{~b}$ shows results obtained using the sensitive modes of the system. In both plots, it is shown that, for the case of zero noise, damage is predicted exactly. For $10 \%$ noise, the actual damage is also predicted accurately. However, the results in Fig. $4 \mathrm{a}$ show that the deviation in the damage locations is significantly larger and there are damages predicted by ROM in two other damage scenarios where there is no damage.

\section{Damage Identification by Hot Spot Projection Versus Minimum Rank Perturbation Theory}

In this section, the differences between DIHSP and MRPT are explored. The results for both methodologies in a case with a $15 \%$ loss of stiffness in beam $4,30 \%$ loss in beam 5, and $20 \%$ loss in beam 7 are plotted in Fig. 5. For 5\% random eigenvector noise, 100 separate numerical simulations were performed and standard deviation error bars are plotted. The $x$ axis in each plot represents the index of a column vector obtained from storing the upper triangular portion of the perturbation stiffness matrix $\Delta \mathbf{K}$ into a column vector. The $y$ axis in the plots represents the entries of the difference $\Delta \mathbf{K}$ between the original and updated stiffness matrices. In both cases, the filtering method that eliminates damage scenarios based on the shift in frequencies was used.

Figure 5a shows results for the case in which MRPT was used to predict damage, whereas Fig. $5 \mathrm{~b}$ shows results for the case in which DIHSP was used. Damage is predicted exactly by each methodology when the noise is zero. For $5 \%$ noise, the results in Fig. 5 show that DIHSP predicts damage more accurately than MRPT. Also, DIHSP predicts fewer false damages elsewhere in the system.

\section{Robustness}

In this section, the robustness of the methodology to damages outside of the hot spots is explored. Figure $\underline{6}$ contains results for a 


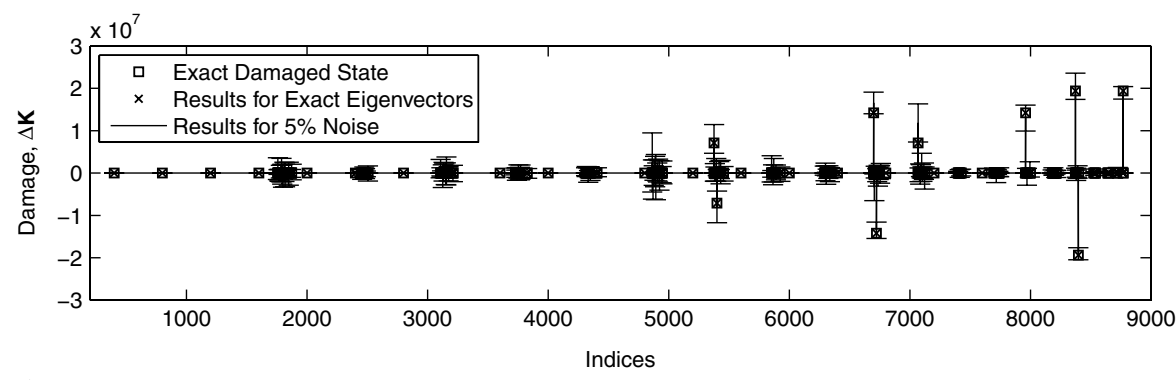

a) MRPT

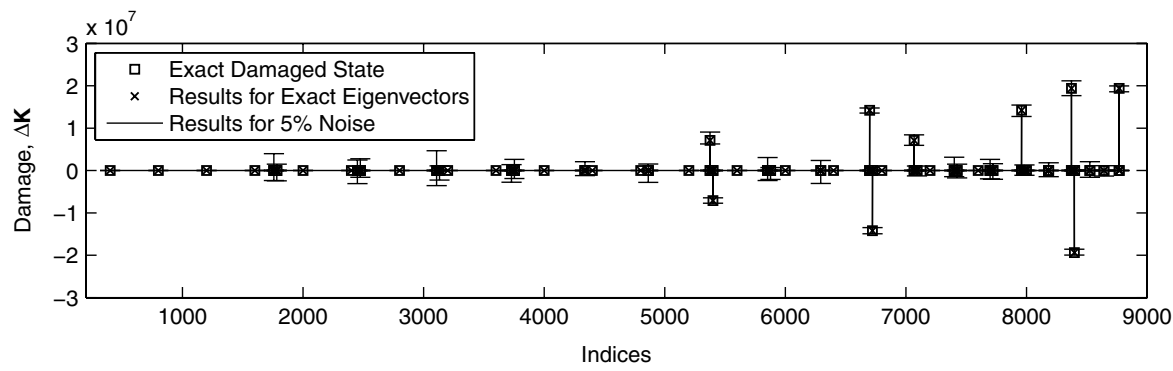

b) DIHSP

Fig. 5 Predicted damage in the linear five-bay frame with damage at three locations and $5 \%$ random eigenvector noise using 20 sensors to find damage using a) MRPT and b) DIHSP.

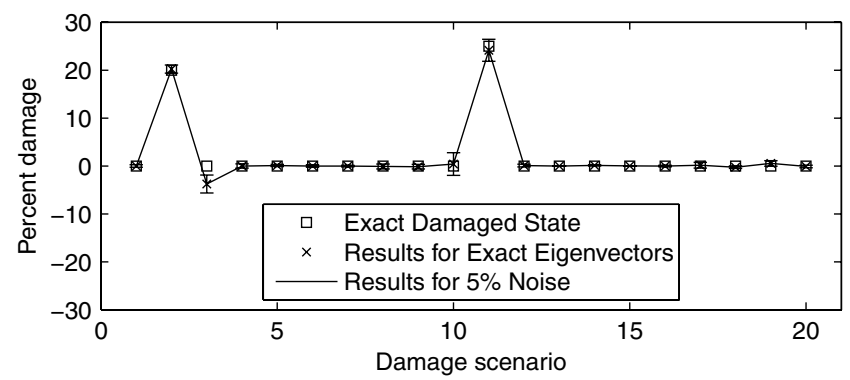

Fig. 6 Predicted damage in the linear five-bay frame with damage in two hot spots and one other location, and $5 \%$ random eigenvector noise using 20 sensors.

$20 \%$ loss of transverse stiffness in beam 1 , and a $25 \%$ loss of transverse stiffness in beam 4 , where these beam numbers are shown in Fig. 2. Additionally, there is $25 \%$ loss of longitudinal stiffness in beam 7 . The last damage was not part of the 20 allowable damage scenarios for the linear system. The plot in Fig. 6 is structured in the same way as in Fig. 4. Standard deviation error bars are plotted for 100 separate numerical simulations in which $5 \%$ random eigenvector noise was added.

Figure 6 shows that, when there is zero noise, exact damage is not predicted $\bar{b}$ ecause there are damages that are occurring in the system at locations assumed undamageable. Although the damages to the system in and out of the hot spots are of a similar level, the method predicts the damage in the hot spots within about $2 \%$ of the actual damage (and DIHSP does not predict any damage outside of the hot spots). Also, very little false damage is predicted in the other damage scenarios. For 5\% random eigenvector noise, the damage is still predicted well with the largest deviation of about $2 \%$ occurring at the third damage scenario.

Figure 7 shows results for a $20 \%$ loss of transverse stiffness in beam 1 , a $\overline{15} \%$ loss in beam 4 , a $30 \%$ loss in beam 5 , and a $25 \%$ loss in beam 7, where these beam numbers are shown in Fig. 2. Additionally, there is $20 \%$ loss of longitudinal stiffness in beam $\overline{1}$, and a $10 \%$ loss of longitudinal stiffness in beam 3 . The latter two damages were not part of the 20 allowable damage scenarios for the linear system. The plot in Fig. 7 is structured in the same way as in Fig. 4. Standard deviation error bars are plotted for 100 separate numerical simulations in which $5 \%$ random eigenvector noise was added.

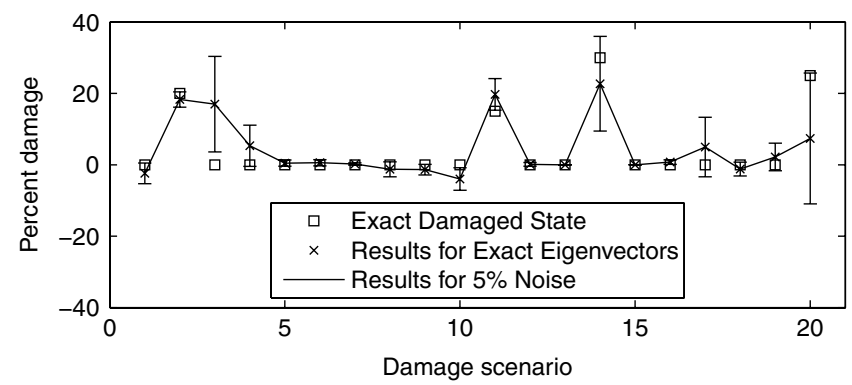

Fig. 7 Predicted damage in the linear five-bay frame with damage in four hot spots and two other locations, and $5 \%$ random eigenvector noise using 20 sensors.

Figure 7 shows that, when there is zero noise, exact damage is not predicted because there are damages that are occurring in the system at locations outside of the assumed hot spots. In this case, the results are not as good as those in Fig. $\underline{6}$ due to the large number of damages that are occurring simultaneously. Although the damage at locations where there is actual damage is predicted relatively accurately in all cases except one, there is a significant amount of false damage predicted in the third, fourth, and 17th damage scenarios in particular.

\section{Filtering}

In this section, the benefit of the filtering algorithm, which eliminates damage scenarios based on the shift in frequencies, is demonstrated.

Figure 8 shows results for a case with a $20 \%$ loss of stiffness in beam 1. The plots in Fig. 8 are structured in the same way as in Fig. 4. Standard deviation error $\bar{b}$ ars are plotted for 100 separate numerical simulations in which $10 \%$ random eigenvector noise was added. Figure 8a shows results for the case without filtering, and Fig. 8b shows results for the case with filtering. When there is zero noise, both cases predict damage exactly. For $10 \%$ noise, both cases predict the damage to a similar level of accuracy, but the results obtained using filtering (Fig. 8b) exhibit less noise in the other damage scenarios (where there is no damage).

\section{Effect of Additional Sensors}

In this section, the benefit of using additional sensors is illustrated. Figure $\underline{9}$ shows results for a case with a $15 \%$ loss of stiffness in 


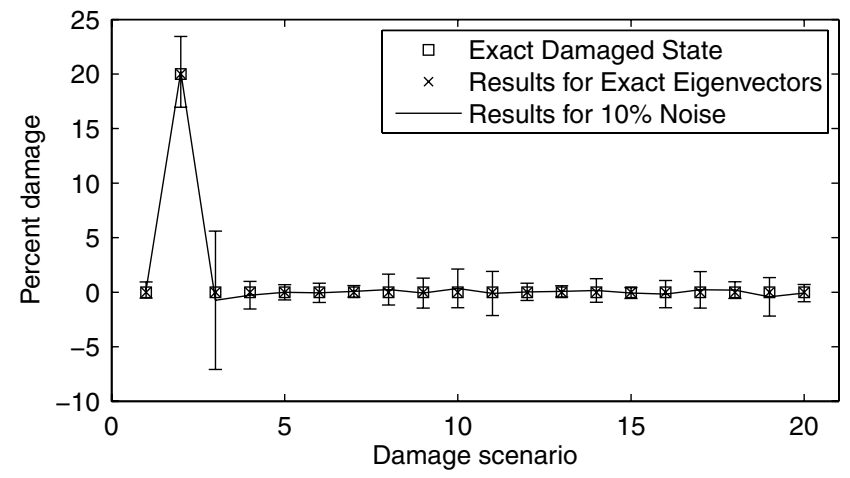

a) Filter off

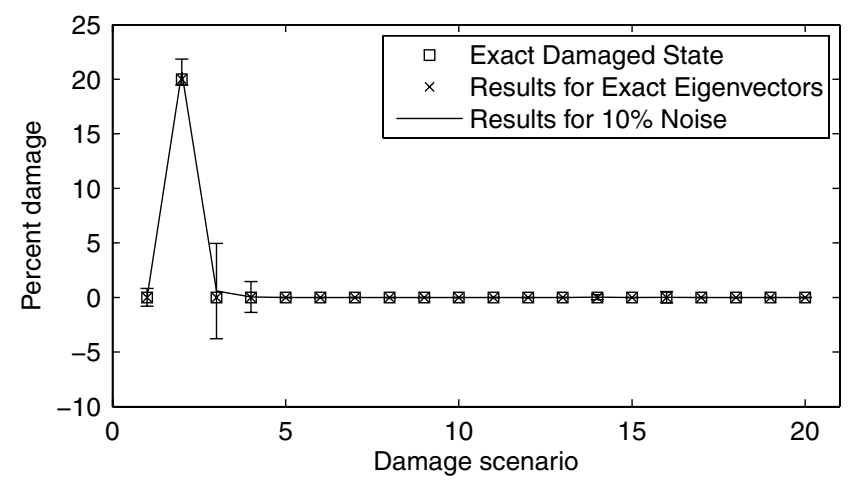

b) Filter on

Fig. 8 Predicted damage in the linear five-bay frame with damage at one location and $10 \%$ random eigenvector noise using 20 sensors a) without the frequency shift filter and b) with the frequency shift filter.

beam 2, 25\% loss in beam 3, 30\% loss in beam 4, and $25 \%$ loss in beam 7. The plots in Fig. 9 are structured in the same way as in Fig. 4. For 5\% random eigenvector noise, 100 separate numerical simulations were performed and standard deviation error bars are plotted. Figure 9a shows results for the case in which 20 sensors are used, and Fig. $\overline{9 b}$ shows results for the case in which 21 sensors are used. The one sensor added to the 20 located at the hot spots was placed using the generalized EIDV method. When there is zero noise, both sensor placements predict damage exactly. For 5\% noise, the 21 sensors (Fig. 9b) predict the damage significantly better in the scenarios with damage, and predict significantly fewer false damages compared with the results obtained using 20 sensors (Fig. 9a).

\section{Nonlinear Five-Bay Structure}

In this section, the use of ROHA and DIHSP is demonstrated for determining damage in linear and nonlinear elements of the nonlinear structure shown in Fig. 3 .

Figure 10 shows results for a case with a $15 \%$ loss of stiffness in plate A, and $20 \%$ loss in plate B (see Fig. 3). The plot in Fig. 10 is structured in the same way as in Fig. 4. Standard deviation error bars are plotted for 100 separate numerical simulations in which 5\% random eigenvector noise was added. When there is zero noise, exact damage is predicted. For $5 \%$ noise, the actual damage is predicted very accurately.

Figure 11 shows the results for a case with a $35 \%$ loss of stiffness in plate $\mathrm{A}, \overline{3} 0 \%$ loss in plate $\mathrm{B}, 30 \%$ loss in beam C, and $25 \%$ loss in beam D (see Fig. 3). The plot in Fig. 11 is structured in the same way as in Fig. 4. Standard deviation error bars are plotted for 100 separate numerical simulations in which $5 \%$ random eigenvector noise was added. When there is zero noise, exact damage is predicted. For 5\% noise, the actual damage is predicted very accurately, with little false damage predicted in other scenarios.

\section{Discussion}

Several new ideas are incorporated into this integrated sensor placement and damage detection methodology. A major aspect of the

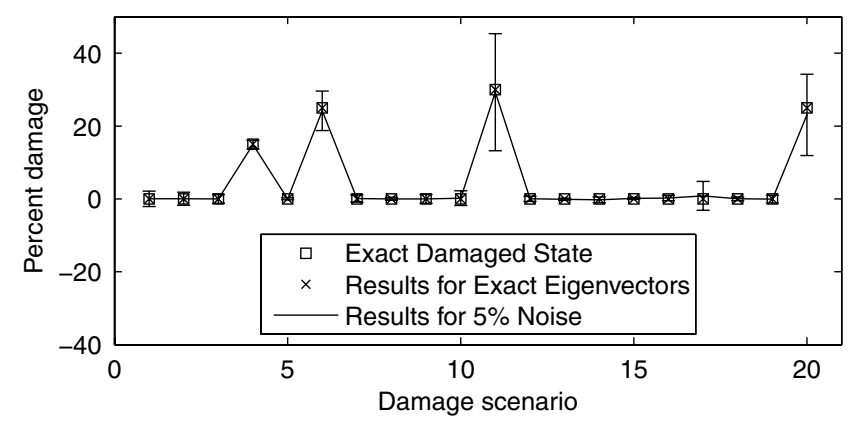

a) Results for 20 sensors

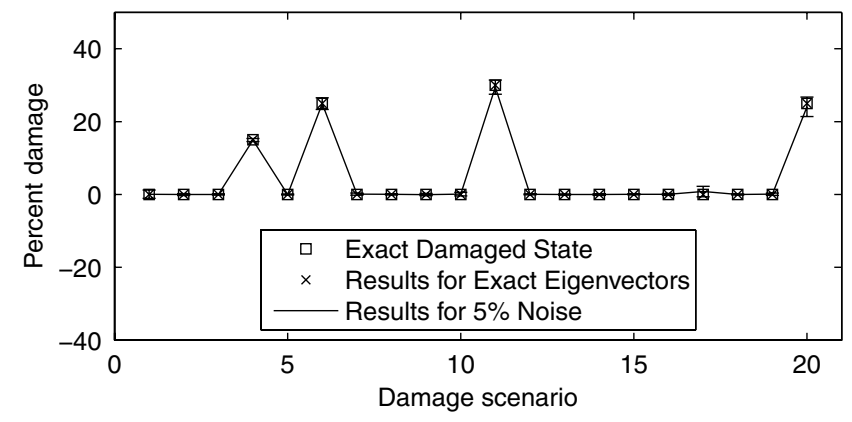

b) Results for 21 sensors

Fig. 9 Predicted damage in the linear five-bay frame with damage at four locations and $5 \%$ random eigenvector noise using a) 20 sensors and b) 21 sensors.

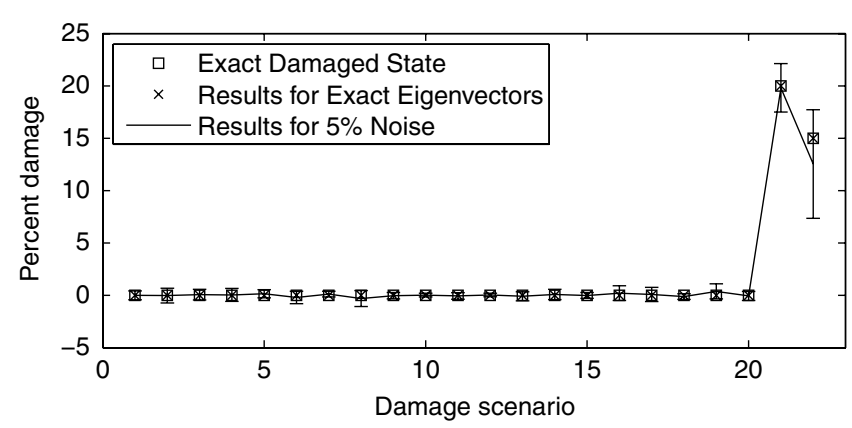

Fig. 10 Predicted damage in the nonlinear five-bay frame with damage in both cubic stiffnesses and $5 \%$ random eigenvector noise using 20 sensors.

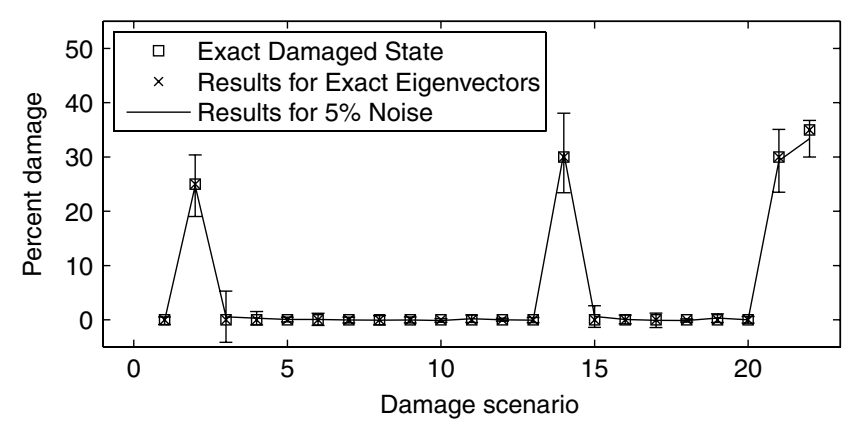

Fig. 11 Predicted damage in the nonlinear five-bay frame with damage in both cubic stiffnesses and 2 linear hot spots, and $5 \%$ random eigenvector noise using 20 sensors.

methodology is ROHA. ROHA differs fundamentally from ROM techniques in its goal. ROM techniques are used to capture the dynamics of the system. Therefore, they use the dominant modes of the system, which tend to be the modes corresponding to the lowest frequencies of the system. In contrast, ROHA is used to characterize changes in the dynamics, and, as a result, employs the modes that are 
sensitive to changes in the hot spots of the system. ROHA uses a frequency-filtering algorithm to remove frequency content of the modes outside the selected modes, so that the partial eigenstructure obtained corresponds to the desired modes. The partial modes are then expanded to the full space using information about the possible damage locations. Other algorithms that expand the modes within the small dimensional space of a set of the healthy eigenvectors fail because damages cause the eigenvectors to vary/rotate into the space of a large number of eigenvectors of the original healthy system.

Another major aspect of the methodology is the improved sensor placement. For the case where the number of sensors equals the number of damageable degrees of freedom, and the hot spots of the system are accessible, the sensors are placed at the hot spots of the system. If additional sensors can be used, a generalized EIDV method can be applied to place these sensors (especially when some hot spots are inaccessible for sensor placement).

EIDV is inherently a quasi-optimal method in that it finds the optimal sensor choice at each step, but all the choices together do not necessarily lead to the global optimum sensor placement. However, using EIDV to place the remaining sensors is effective because EIDV searches for the optimal locations of the remaining sensors only.

The DIHSP technique is another important component of the methodology. The key advantage DIHSP has over other modalbased techniques is that it filters out all damages except the desired damage scenarios. This is also why DIHSP is only truly useful when the damage scenarios are known in advance.

The final novel aspect of the methodology herein is that it was extended to nonlinear (augmented) systems. When applied to nonlinear systems, the methodology as a whole is essentially the same as for linear systems. The only difference is that using nonlinear ROHA/DIHSP requires the measurement of the degrees of freedom that contain nonlinearities. This is necessary to form the augmented equations of motion.

\section{Conclusions}

A method to place sensors for damage detection in linear and nonlinear systems has been presented. The sensor placement approach is based on determining the eigenvectors most sensitive to changes in damageable hot spots in the system. The full modes are extracted from partial measurements by using a novel reduced-order health assessment technique. Damage can then be assessed using any number of modal-based approaches or by damage identification by hot spot projection. Nonlinear systems can be handled using this methodology by exploiting the features of augmented linear systems. The algorithms proposed have been explored numerically for linear and nonlinear structures. The effectiveness of the proposed methods were demonstrated, and the effects of measurement errors were presented.

\section{Acknowledgment}

The authors wish to acknowledge the National Science Foundation (CAREER and Graduate Research Fellowship programs) for the generous support of this work.

\section{References}

[1] Cherng, A., "Optimal Sensor Placement for Modal Parameter Identification Using Signal Subspace Correlation Techniques," Mechanical Systems and Signal Processing, Vol. 17, No. 2, 2003, pp. 361-378. doi:10.1006/mssp.2001.1400

[2] Yuen, K., Katafygiotis, L. S., Papadimitriou, C., and Mickleborough, N. C., "Optimal Sensor Placement Methodology for Identification with Unmeasured Excitation," Journal of Dynamic Systems, Measurement, and Control, Vol. 123, No. 4, 2001, pp. 677-686. doi:10.1115/1.1410929

[3] Penny, J.E. T., Friswell, M. I., and Garvey, S. D., "Automatic Choice of Measurement Locations for Dynamic Testing," AIAA Journal, Vol. 32, No. 2, 1994, pp. 407-414.

doi: $10.2514 / 3.11998$

[4] Cobb, R. G., and Liebst, B. S., "Sensor Placement and Structural
Damage Identification from Minimal Sensor Information," AIAA Journal, Vol. 35, No. 2, 1997, pp. 369-374.

[5] Shi, Z. Y., Law, S. S., and Zhang, L. M., "Optimum Sensor Placement for Structural Damage Detection," Journal of Engineering Mechanics, Vol. 126, No. 11, 2000, pp. 1173-1179. doi:10.1061/(ASCE)0733-9399(2000)126:11(1173)

[6] Guo, H. Y., Zhang, L., Zhang, L. L., and Zhou, J. X., "Optimal Placement of Sensors for Structural Health Monitoring Using Improved Genetic Algorithms," Smart Materials and Structures, Vol. 13, No. 3 , 2004, pp. 528-534. doi:10.1088/0964-1726/13/3/011

[7] Shi, Z. Y., Law, S. S., and Zhang, L. M., "Damage Detection by Directly Using Incomplete Mode Shapes," Journal of Engineering Mechanics, Vol. 126, No. 6, 2000, pp. 656-660. doi:10.1061/(ASCE)0733-9399(2000)126:6(656)

[8] Bazoune, A., Khulief, Y. A., Stephen, N. G., and Mohiuddin, M. A., "Dynamic Response of Spinning Tapered Timoshenko Beams Using Modal Reduction," Finite Elements in Analysis and Design, Vol. 37, No. 3, 2001, pp. 199-219. doi:10.1016/S0168-874X(00)00030-5

[9] Bladh, R., Castanier, M. P., and Pierre, C., "Reduced Order Modeling and Vibration Analysis of Mistuned Bladed Disk Assemblies with Shrouds," Journal of Engineering for Gas Turbines and Power, Vol. 121, No. 3, 1999, pp. 515-522. doi: $10.1115 / 1.2818503$

[10] Bladh, R., Castanier, M. P., and Pierre, C., "Component-Mode-Based Reduced Order Modeling Techniques for Mistuned Bladed Disks, Part 2: Application," Journal of Engineering for Gas Turbines and Power, Vol. 123, No. 1, 2001, pp. 100-108. doi:10.1115/1.1338948

[11] Castanier, M. P., Tan, Y. C., and Pierre, C., "Characteristic Constraint Modes for Component Mode Synthesis," AIAA Journal, Vol. 39, No. 6 , 2001, pp. 1183-1187.

[12] Epureanu, B. I., Hall, K. C., and Dowell, E. H., "Reduced Order Models of Unsteady Transonic Viscous Flows in Turbomachinery," Journal of Fluids and Structures, Vol. 14, No. 8, 2000, pp. 1215-1235. doi: $10.1006 /$ jfls. 2000.0320

[13] Epureanu, B. I., Hall, K. C., and Dowell, E. H., "Reduced Order Models of Unsteady Viscous Flows in Turbomachinery Using Viscous-Inviscid Coupling," Journal of Fluids and Structures, Vol. 15, No. 2, 2001, pp. 255-276. doi:10.1006/jfls.2000.0334

[14] Farrell, B. F., and Ioannou, P. J., "Accurate Low-Dimensional Approximation of the Linear Dynamics of Fluid Flow," Journal of Atmospheric Sciences, Vol. 58, No. 18, 2001, pp. 2771-2789.

[15] Zimmerman, D. C., "Model Validation and Verification of Large and Complex Space Structures," Inverse Problems in Engineering, Vol. 8, No. 2, 2000, pp. 93-118. doi: $10.1080 / 174159700088027722$

[16] Zimmerman, D. C., "Looking into the Crystal Ball: The Continued Need for Multiple Viewpoints in Damage Detection," Key Engineering Materials: Damage Assessment of Structures, Vols. 167-168, No. 1, 1999, pp. 76-90.

[17] Zimmerman, D. C., and Simmermacher, T., "Model Correlation Using Multiple Static Load and Vibration Tests," AIAA Journal, Vol. 33, No. 11,1995 , pp. 2182-2188. doi: $10.2514 / 3.12813$

[18] Zimmerman, D. C., and Kaouk, M., "Structural Damage Detection Using Minimum Rank Update Theory," Journal of Vibration and Acoustics, Vol. 116, No. 2, 1994, pp. 222-231. doi: $10.1115 / 1.2930416$

[19] Liu, P. L., "Identification and Damage Detection of Trusses Using Modal Data," Journal of Structural Engineering, Vol. 121, No. 4, 1995, pp. 599-608. doi:10.1061/(ASCE)0733-9445(1995)121:4(599)

[20] Kim, H. S., and Chun, Y. S., "Structural Damage Assessment of Building Structures Using Dynamic Experimental Data," Structural Design of Tall and Special Buildings, Vol. 13, No. 1, 2004, pp. 1-8. doi:10.1002/tal.227

[21] D'Souza, K., and Epureanu, B. I., "Damage Detection in Nonlinear Systems Using System Augmentation and Generalized Minimum Rank Perturbation Theory," Smart Materials and Structures, Vol. 14, No. 5, 2005, pp. 989-1000. doi:10.1088/0964-1726/14/5/037

[22] D'Souza, K., and Epureanu, B. I., "Multiple Augmentations of Nonlinear Systems and Generalized Minimum Rank Perturbations for Damage Detection," Journal of Sound and Vibration, Vol. 316, Nos. 15, 2008, pp. 101-121. doi:10.1016/j.jsv.2008.02.018 
[23] Leuridan, J., "Some Direct Parameter Model Identification Methods Applicable for Multiple Modal Analysis," Ph.D. Thesis, Dept. of Mechanical and Industrial Engineering, Univ. of Cincinnati, Cincinnati, OH, 1984.

[24] Hung, C., Ko, W., and Peng, Y., "Identification of Modal Parameters from Measured Input and Output Data Using a Vector Backward Auto-
Regressive with Exogeneous Model," Journal of Sound and Vibration, Vol. 276, Nos. 3-5, 2004, pp. 1043-1063.

doi:10.1016/j.jsv.2003.08.020 